\title{
RDUS
}

Revue de DROIT UNIVERSITÉ DE SHERBROOKE

Titre : $\quad$ LA CONFIANCE, INSTRUMENT DE RÉGULATION DES ENVIRONNEMENTS ÉLECTRONIQUES

Auteur(s): $\quad$ Cynthia CHASSIGNEUX

Revue : $\quad$ RDUS, 2006-2007, volume 37, numéro 2

Pages: $\quad 441-472$

ISSN : $\quad 0317-9656$

Éditeur : $\quad$ Université de Sherbrooke. Faculté de droit.

URI : $\quad$ http://hdl.handle.net/11143/11840

DOI : 
Page vide laissée intentionnellement. 


\title{
ARTICLE
}

\section{LA CONFIANCE, INSTRUMENT DE RÉGULATION DES ENVIRONNEMENTS ÉLECTRONIQUES}

\author{
par Cynthia CHASSIGNEUX**
}

Dans un environnement où les acteurs ne sont pas en présence l'un de l'autre, la confiance joue un rôle important. Toutefois quels en sont les fondements définitionnels, comment catégoriser la relation s'établissant entre l'émetteur et le destinataire de celle-ci et comment se matérialise-t-elle. Telles sont les avenues envisagées par l'auteure afin de démontrer que la confiance est un instrument de régulation des environnements électroniques.

In an environment where the parties are not always in each others' presence, trust necessarily plays an important role. However, what are the underpinnings defining this relationship, how should the relationship between the issuer and the addressee be characterized, and finally, how does it materialize? These are issues discussed by the writer who seeks to demonstrate that trust can be a means of regulating the electronic environment.

* $\quad$ Cet article s'inscrit dans le cadre d'une recherche post-doctorale sur les mécanismes de production de la confiance à l'égard de la protection des renseignements personnels dans les environnements électroniques, financée par le Centre de recherche en éthique de l'Université de Montréal (CRÉUM). L'auteure tient à remercier MM. Jean-Guy Belley, Ejan Mackaay et Pierre Trudel pour leurs précieux commentaires sur les versions antérieures de ce texte.

** Chercheure post-doctorale. Docteure en droit de l'Université PanthéonAssas (Paris II) et de l'Université de Montréal. Agente de recherche au Centre de recherche en droit public (CRDP) de l'Université de Montréal. Chargée de cours à l'Université de Montréal et de Sherbrooke. 


\section{SOMMAIRE}

Introduction

La confiance, une notion aux multiples fondements définitionnels

La confiance, une notion aux multiples catégories 463

La confiance, une notion aux multiples matérialisations ... 467

Conclusion .................................................... 471 


\section{Introduction}

Le recours aux technologies de l'information et de la communication dans les activités de la vie politique, économique et sociale doit s'effectuer dans le respect des droits de tout un chacun. Ces droits sont majoritairement énoncés dans des textes réglementaires, édictés et sanctionnés par la puissance publique ${ }^{1}$. Ils sont également contenus dans des instruments autoréglementaires ${ }^{2}$, volontairement mis en place par des entités, souvent de nature privée, oeuvrant dans et pour un secteur donné. Se profile ici l'idée de réseau, de communauté. Ils sont aussi décrits dans des approches de co-régulation ${ }^{3}$ regroupant des entités des secteurs public et privé travaillant pour un même

1. Pour une analyse générale du mécanisme réglementaire appliqué aux environnements électroniques, voir notamment: Pierre Trudel, France Abran, Karim Benyekhlef et Sophie Hein, Droit du cyberespace, Montréal, Thémis, 1997 aux pp. 3-13 à 3-32 [Trudel, Abran, Benyekhlef et Hein, "Cyberespace»].

2. Pour une analyse générale du mécanisme d'autoréglementation appliqué aux environnements électroniques, voir notamment Trudel, Abran, Benyekhlef et Hein, "Cyberespace", supra note 1 aux pp. 3-34 à 3-46; Pierre Trudel, "Les effets juridiques de l'autoréglementation" (1989) 19 R.D.U.S. 247.

3. Pour une illustration de cette approche, il est possible de consulter des organismes tels que le Forum des droits sur Internet (France) : Forum des droits sur Internet <http://www.foruminternet.org>. L'idée d'une telle approche est apparue nécessaire à la suite du rapport Internet et les réseaux numériques rendu par le Conseil d'État en 1998 : "Internet et les réseaux numériques" (1998), en ligne: Le Conseil d'État <http:// www.conseil-etat.fr/ce/rappor/index_ra_liau01.shtml>. Le Forum des droits sur Internet fait partie des organismes constituant le Réseau européen de corégulation de l'Internet : Réseau européen de corégulation de l'Internet <http://network.foruminternet.org>. dont est membre l'Observatoire des Droits de l'Internet (Belgique) : Observatoire des Droits de l'Internet <http://www.internet-observatory.be>, qui a rendu, en 2004, un avis visant à renforcer la confiance dans le commerce électronique : "Observatoire des Droits de l'Internet, Avis n³ - Pistes pour renforcer la confiance dans le commerce électronique" (Juin 2004), en ligne: Observatoire des Droits de l'Internet <http://www.internetobservatory.be/internet_observatory/pdf/advices/advice_fr_003.pdf>. 
La confiance,

instrument de régulation

(2007) 37 R.D.U.S. des instruments électroniques

objectif : encadrer adéquatement les opérations effectuées sur ce nouveau territoire qu'est Internet ${ }^{4}$.

Cette concurrence, ou plutôt cette coexistence des normes ${ }^{5}$ s'explique notamment par la nature transfrontalière et décentralisée d'Internet, par sa technicité, par la fluidité et la fugacité des informations qui y circulent. Elle a pour conséquence que, face à la lenteur et à la complexité des rouages parlementaires 6 , les acteurs de ce médium se dotent de mécanismes visant à encadrer les activités qui s'y déroulent tant dans le secteur public que privé7. On assiste à un bouleversement des conceptions classiques. On passe, en effet, d'une conception moniste du droit à une approche plurielle ${ }^{8}$ de son entendement.

4. Yves Poullet, "Technologies de l'information et de la communication et "co-régulation" : une nouvelle approche?" (26 mai 2004), en ligne : Droit et Technologies <http://www.droit-technologie.org>.

5. Michel Vivant, "Le futur des relations entre le droit et les technologies de l'information" (2002) 20 Cahiers du Centre de recherches informatique et droit 61.

6. Vincent Gautrais, Guy Lefebvre et Karim Benyekhlef, "Droit du commerce électronique et normes applicables: l'émergence de la lex electronicas (1997) 5 R.D.A.I. 547.

7. Pour une illustration de ce phénomène voir notamment Thomas Schultz, "eBay: un système juridique en formation?" (2005) 22 Revue du Droit des Technologies de l'Information 27 [Schultz, "eBay»].

8. Pour un regard théorique de cette approche plurielle du droit, voir notamment Jacques Chevallier, "L'ordre juridique» dans Le droit en procès, Paris, P.U.F., 1984 aux pp. 7 à 49; Jean-Guy Belley (dir.), Le droit soluble: contributions québécoises à l'étude de l'internormativité, Paris, LGDJ, 1996; Andrée Lajoie, "Synthèse introductive" dans Andrée Lajoie, Jean-Maurice Brisson, Sylvio Normand et Alain Bissonnette, dir., Le statut juridique des peuples autochtones au Québec et le pluralisme, Montréal, Yvon Blais, 1996 aux pp. 7 à 19; Jean-Guy Belley, "L’État et la régulation juridique des sociétés globales. Pour une problématique du pluralisme juridique" (1986) XVIII-1 Sociologie et Sociétés 27; John Griffith, "What is legal pluralism" (1986) 24 J. Legal Pluralism 1; Roderick A. MacDonald, "Pour la reconnaissance d'une normativité juridique implicite et "inférentielle" (1986) 28 (1) Sociologie et sociétés 47; Pierre Lascoumes et Evelyne Serverin, "Le droit comme activité sociale : pour une approche wébérienne des activités juridiques" (1988) 9 Droit et société 165; Guy Rocher, "Pour une sociologie des ordres juridiques" (1988) 29 C. de D. 91 [Rocher, "Sociologie»]; Gérard Timsit, "Sur 
Cette transformation du droit, soulignée par une analyse "post-moderne" du droit ${ }^{9}$ ou encore par l'effet d'un changement de paradigme ${ }^{10}$, conduit à envisager l'encadrement des activités effectuées par le biais des environnements électroniques non plus au regard du seul droit positif mais de l'ensemble des mécanismes développés à cet effet, compte tenu du fait que :

[L]a compréhension du cadre juridique d'une activité ne peut découler uniquement d'une vision linéaire du phénomène juridique. Il faut envisager les autres normes, celles qui ne sont pas considérées comme juridiques par la plupart des juristes, mais qui ont un effet normatif incontestable. ${ }^{11}$

$\mathrm{Au}$ fil des ans, cette "nouvelle" approche du droit s'est illustrée par le développement d'une kyrielle d'instruments accompagnant l'implantation du paiement en ligne, des prestations électroniques de service, de l'informatisation du secteur de la santé et des services sociaux, des modes alternatifs de règlement des conflits, du vote électronique, du transfert de renseignements personnels aussi bien à des fins commerciales que de sécurité aux frontières, pour ne citer que ces exemples. Parmi ces instruments, il est généralement fait référence aux codes de conduite, à la certification, aux labels de qualité ${ }^{12}$, aux

l'engendrement du droit" (1988) Revue de Droit Public 39; Andrée Lajoie, "Contribution à une théorie de l'émergence du droit. I - Le droit, l'État, la société civile, le public, le privé : de quelques définitions interreliées" (1991) 25 R.J.T. 103; Jacques Vanderlinden, "Vers une nouvelle conception du pluralisme juridique" (1993) 2 R.R.J. 573.

9. Pour une illustration de cette approche, voir notamment: Jacques Chevallier, L'État post-moderne, Paris, L.G.D.J., 2003.

10. Pour une illustration de cette approche, voir notamment : François Ost et Michel Van de Kerchove, De la pyramide au réseau? Pour une théorie dialectique du droit, Bruxelles, Publications des Facultés universitaires Saint-Louis, 2002.

11. Trudel, Abran, Benyekhlef et Hein, "Cyberespace», supra note 1 à la p. 31.

12. Pour une analyse du processus de labelisation et de certification appliqué aux environnements électroniques, voir notamment Cynthia 
guides $^{13}$ ou modèles de pratique ${ }^{14}$, aux politiques de confidentialité $^{15}$, à la régulation par l'architecture ${ }^{16}$, aux best

Chassigneux, Vie privée et commerce électronique, Montréal, Thémis, 2005 aux pp. 201 et s. [Chassigneux, "Vie privée»].

13. Pour une illustration de cette méthode, voir les différents guides développés par la Chaire L. R. Wilson sur le droit des technologies de l'information et du commerce électronique, entre autres dans les domaines suivants : administration électronique, santé, scolaire. En ligne : Chaire Wilson <http://www.chairelrwilson.ca.

14. Pour une illustration de cette méthode, voir Québec, Ministère des relations avec les citoyens et l'immigration, Direction du soutien en accès à l'information et en protection des renseignements personnels, Modèle de pratique de protection des renseignements personnels. Dans le contexte du développement des systèmes d'information par les organismes publics, Sainte-Foy, Publications du Québec, 2004.

15. Pour une analyse des politiques de confidentialité, voir notamment Chassigneux, "Vie privée", supra note 12 aux pp. 178 et s. À ce sujet, il est également possible de consulter le "Générateur de l'OCDE de déclaration de protection de la vie privée», en ligne: OCDE <http://www.oecd.org/document/42/0,2340,fr_2649_34255_28879786_ 1_1_1_ 1,00.html> fournissant: "[...] des conseils pour procéder, à l'intérieur de l'organisation, à l'examen des pratiques en matière de protection de la vie privée, et pour élaborer une déclaration de politique de protection de la vie privée".

16. L'idée de régulation par l'architecture est particulièrement présente sous la plume de Joël R. Reidenberg, Graham Greenleaf et Lauwrence Lessig, dont les écrits respectifs, par exemple, sont: Joël R. Reidenberg, "Governing Networks and Rule-Making in Cyberspace" (1996) 45 Emory L.J. 911; Joël R. Reidenberg, "Lex Informatica: The Formulation of Information Policy Rules Through Technology" (1998) 76-3 Tex. L. Rev.; Graham Greenleaf, "An Endnote on Regulating Cyberspace: Architecture vs. Law», en ligne : (1998) U.N.S.W.L.J. 52 <http://www.austlii.edu.au/ au/journals/UNSWLJ/1998/52.html>; Lawrence Lessig, "The Law of the Horse: What Cyberlaw Might Teach" (1999) 113 Harv. L. Rev. 501; Lawrence Lessig, Code and other laws of cyberspace, New York, Basic Book, 1999 [Lessig, "Code"]; David G. Post, "Anarchy, State, and the Internet: An Essay on Law-Making in Cyberspace», en ligne : (1995) Journal of Online Law 3 <http://www.cli.org/DPost/ X0023_ANARCHY.html>. Voir également, Yves Poullet, "La technologie et le droit : du défi à l'alliance" dans Mélanges Guy Horsmans, Bruxelles, Bruylant, 2004, 943; Thierry Boidart et Lilianne Esnault, "Les autoroutes de l'information et les ambivalences de la technologies" dans Mélanges Roger Delay Thermoz, Paris, Eska, 1995, 65. 
practices et autres contrats ${ }^{17}$.

Ces mécanismes, en plus de prescrire les droits et obligations de chacun, ont pour objectif d'établir un lien de confiance entre l'administration et le citoyen, entre le commerçant électronique et l'internaute, entre deux entités juridiques. Ce lien, qui doit prévaloir à toute relation peu importe sa nature, est particulièrement indispensable dans un environnement où les parties ne sont pas en présence l'une de l'autre, ne sont pas sur le même territoire ${ }^{18}$.

Ainsi :

[S]i les hommes doivent traiter avec des appareils bureaucratiques devenus autonomes, s'ils doivent gérer des relations avec un Autrui absent, ou éloigné, avec lequel ils ne sont plus en face à face, s'ils doivent se contenter d'informations parcellaires sur leur avenir, ou si ce dernier devient hasardeux, la confiance devient un médium fort utile. Et l'auteur de continuer par une citation de Giddens ${ }^{19}$

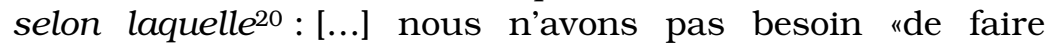
confiance à quelqu'un dont les activités sont visibles en permanence et dont les modes de raisonnements sont transparents, ni de faire confiance à un système dont les rouages sont parfaitement connus et compris». ${ }^{21}$

17. Pour une analyse générale de la pratique contractuelle appliquée aux environnements électroniques, voir notamment Trudel-AbranBenyekhlef-Hein, "Cyberespace", supra note 1 aux pp. 3-33 à 3-34. Pour une approche didactique de cette problématique à l'intention des cyberconsommateurs français et québécois, voir la Carte des recours $d u$ cyberconsommateur: "Carte des recours du cyberconsommateur", en ligne : Cyberconsommation <http://www.cyberconsommation.ca>.

18. Voir notamment, OCDE, Groupe de travail sur les indicateurs pour la société de l'information, Étude exploratoire pour la mesure de la confiance dans l'environnement en ligne, $\mathrm{n}^{\circ}$ de doc. DSTI/ICCP/IIS(2005)1/FINAL (2005) [OCDE, "Mesure confiance»].

19. Anthony Giddens, Les conséquences de la modernité, Paris, L'Harmattan, 1994 [Giddens, "Modernité»].

20. Ibid. à la p. 140 .

21. Christian Thuderoz, "Introduction au propos : la confiance en question" dans Vincent Mangematin et Christian Thuderoz, dir., Des mondes de 
Cette notion de confiance qui "a longtemps semblé appartenir à un registre désuet, non moderne» 22 est (re)mise sur le devant de la scène depuis quelques années, dans la littérature sociologique, politique, économique, juridique, cherchant à comprendre le déroulement des activités réalisées dans le cadre des environnements électroniques ${ }^{23}$, sur Internet, par exemple. Pour s'en rendre compte il suffit de se référer aux livres ${ }^{24}$, articles de revue ${ }^{25}$, lois ${ }^{26}$,

confiance. Un concept à l'épreuve de la réalité sociale, Paris, CNRS Éditions, 2003, 19 à la p. 26 [Thuderoz, "Introduction au propos»].

22. Christian Thuderoz, "Introduction générale: pourquoi interroger la notion de confiance?" dans Christian Thuderoz, Vincent Mangematin et Denis Harrison, dir., La confiance. Approches économiques et sociologies, Paris, Gaétan Morin Éditeur Europe, 1999, 1 à la p 1.

23. Denis Harrisson, "Confiance identitaire, confiance cognitive et processus d'innovation" dans Christian Thuderoz, Vincent Mangematin et Denis Harrison, dir., La confiance. Approches économiques et sociologies, Paris, Gaétan Morin Éditeur Europe, 1999, 209: l'auteur insiste, en introduction, sur le fait que : "L'engouement actuel pour le thème de la confiance témoigne d'un renouvellement des relations sociales qui questionne son rôle. En effet, les préoccupations des agents portent sur d'autres aspects relationnels que la confiance lorsque celle-ci est considérée comme étant acquise dans les relations sociales institutionnelles. Il suffit de proposer de nouvelles avenues relationnelles pour qu'aussitôt la confiance resurgisse comme un élément a priori, sans lequel les agents ne peuvent poursuivre leur échange».

24. Voir, entre autres, Séverine Mas-Foveau et Malika Benachour-Verstepen, Le commerce électronique en toute confiance. Diagnostic des pratiques et environnement juridique, Paris, Litec, 2001; Magda Fusaro, Commerce électronique. Comment créer la confiance, Montréal, Isabelle Quentin Éditeur, 2002.

25. Voir notamment, Danielle Stanton, "TIC et éthique : rétablir la confiance" (2004) Centre Francophone d'informatisation des organisations Perspectives 22.

26. Voir notamment, Loi $n^{\circ} 2004-575$ du 21 juin 2004 pour la confiance dans l'économie numérique, J.O., 22 juin 2004, 11168 [Loi pour la confiance dans l'économie numérique]. Cette loi transpose en droit français la Directives CE, Directive 2000/31/CE du Parlement européen et du Conseil du 8 juin 2000 relative à certains aspects juridiques des services de la société de l'information, et notamment du commerce électronique, dans le marché intérieur, [2003] J.O. L 178 aux pp. 0001 - 0016. Pour une analyse critique de cette loi, voir entre autres, Michel Vivant, "Entre ancien et nouveau, une quête désordonnée de confiance pour l'économie numérique" (2004) 171 Lamy Droit de l'informatique et des réseaux 2-20. 
rapports $^{27}$, allocutions ${ }^{28}$ empruntant le chemin de la confiance pour décrire les avenues à retenir en matière de commerce électronique ${ }^{29}$, par exemple.

Toutefois, les contributions de nature juridique ne renseignent pas, ou très peu, sur les fondements, les caractéristiques de la notion de confiance, cette notion étant davantage analysée par des philosophes ${ }^{30}$, des

Par ailleurs, au sujet de la Loi pour la confiance dans l'économie numérique, certains praticiens notent que : "le concept de confiance en réalité, reste un domaine encore peu exploré des juristes. Il serait sans doute judicieux d'en déterminer les contours théoriques. Et sur un plan pratique, d'en préciser les applications", Éric A. Caprioli, "La confiance dans les communications électroniques" (mai 2005), en ligne : Caprioliavocats.com <http://www.caprioli-avocats.com/pages/publications / edocs/commerce_electroniq/edocs_ecommerce_communicationselectroni q.htm>.

27. Voir notamment, France, Exécutive, Ministère de l'économie et de l'industrie, L'e-commerce et la confiance. Sceaux-Certification des sites de commerce électronique - Codes de conduite et Règlement alternatif des litiges, Rapport du Groupe de Travail $n^{\circ} 4.2$ (15 novembre 2001).

28. Yves Poullet et Mireille Antoine, "Vers la confiance ou comment assurer le développement du commerce électronique», allocution présentée lors du Colloque international "L'Internet et le Droit - Droit européen et comparé de l'Internet", Paris, 25 et 26 septembre 2000; Jennifer Stoddart, "Promouvoir le programme d'instauration de la confiance : protection de la vie privée et sécurité - Du commerce électronique à la cyber-économie : stratégie pour le XIXe siècle", allocution de la Commissaire à la vie privée du Canada, Ottawa, 28 septembre 2004.

29. Dans le cadre de cet article, seule la relation s'établissant entre un commerçant et un consommateur sera envisagée. Ne seront donc pas prises en considération les questions inhérentes à l'établissement de la confiance dans le cadre d'une relation entre professionnels ou entre consommateurs.

30. Des auteurs, comme Robert Damien, nuancent cette idée compte tenu du fait que : "La recherche philosophique ne peut en effet, en première approche que témoigner face à la notion de confiance d'un double sentiment: d'une part le sentiment d'être démuni et d'autre part le sentiment d'être submergé. Démuni tout d'abord: la confiance si elle appartient bien au langage courant, n'est pas un concept central dans la pensé philosophique moderne. [...] Mais ce silence est moins une absence qu'une disparition ou un effacement, elle est moins une ignorance conceptuelle qu'un retrait d'investissement. D'où notre deuxième 
sociologues $^{31}$, des économistes ${ }^{32}$. De plus, cet exercice n'est pas chose facile car il existe autant de définitions qu'il y a d'auteurs qui se sont intéressés à cette notion polysémique qui évolue avec le temps ${ }^{33}$ et qui diffère d'un espace géographique à un autre. Les valeurs et les représentations étant multiples cela a une influence sur la dimension de la confiance ${ }^{34}$.

Cette situation permet, possiblement, d'expliquer pourquoi les auteurs, ou le législateur, au lieu de préciser ce qu'ils entendent par cette notion insistent plutôt sur les mécanismes qui permettent de renforcer la sécurité des diverses opérations administratives, commerciales ou personnelles - effectuées dans un environnement dématérialisé. Sont alors abordées les

sentiment, celui d'être dès lors submergé car la notion de confiance se découvre rétrospectivement et au contraire comme un concept central jouant un rôle majeur voire générateur dans deux systèmes de pensée constitutifs de la civilisation occidentale [soit la pensée antique et la pensée chrétienne]". Robert Damien, "La confiance et ses crises philosophiques" dans Romain Laufer et Magali Orillard, dir., La confiance en question, Paris/Montréal, L'Harmattan, 2000, 19 aux pp. 19 et 20 [Damien, "Crises philosophiques"].

31. Voir notamment, Giddens, "Modernité", supra note 19; Nikhlas Luhmann, La confiance. Un mécanisme de réduction de la complexité sociale, Paris, Economica, 2006 (pour la traduction française) [Luhmann, "Confiance»].

32. Pour une analyse économique de la confiance, voir notamment, PierreHugues Vallée et Ejan Mackaay, "La confiance. Sa nature et son rôle dans le commerce électronique», en ligne : (2006) 11 (Automne/Fall 2006) Lex Electronica 2 <http://www.lex-electronica.org/articles/v112/vallee_mackaay.htm> [Vallée et Mackaay, "Confiance"].

33. Pour une illustration de l'évolution de la notion à travers les âges, voir notamment: Damien, "Crises philosophiques", supra note 30 aux pp. 19 à 36.

34. Voir notamment, Alain Peyrefitte, La société de confiance. Essai sur les origines et la nature du développement, Paris, Éditions Odile Jacob, 1995 aux pp. 355 et s. [Peyrefitte, "Développement»]; Francis Fukuyama, La confiance et la puissance. Vertus sociales et prospérité économique, Paris, Plon, 1997 [Fukuyama, "Confiance et puissance»]; Sirkka L. Jarvenpaa et Noam Tractinsky, "Consumer Trust in an Internet Store: A CrossCultural Validation", en ligne: (1999) Journal of Computer-Mediated Communication 5(2) <http://jcmc.indiana.edu/vol5/issue2/jarvenpaa. html>. 
problématiques relatives, notamment, au droit de la preuve, à la signature électronique, au rôle des autorités de certification ou "tiers de confiance", à la sécurité ${ }^{35}$, à la responsabilité36, à la protection des renseignements personnels.

Sans remettre en cause cette orientation de la doctrine juridique, souvent tournée du côté du destinataire de la confiance, c'est-à-dire la puissance publique ou le commerçant, il n'en demeure pas moins que se pose toujours la question de la confiance. Ainsi, le citoyen/consommateur, soit l'émetteur de cette confiance peut se demander : d'où vient-elle? Que signifie-t-elle? Comment s'exprime-t-elle? Comment la qualifier? Comment la reconnaître? Pourquoi l'accorder?

Il serait possible de multiplier à l'infini les interrogations à son égard tant il s'agit d'une notion offrant différentes facettes. Notre propos dans cet article n'est pas de répondre à l'ensemble de ces questions. Notre propos est plutôt exploratoire et consistera à dresser un portrait général de la notion nous aidant à démontrer que la confiance est un instrument de régulation des environnements électroniques, d'Internet, à l'instar d'auteurs économistes pour la plupart - qui se sont intéressés à cette notion

35. Pour une analyse de la confiance au regard du thème de la sécurité, voir notamment: OCDE, "Mesure confiance", supra note 18. Voir également, Francis Mer, "Texte $n^{\circ} 528$ relatif au Projet de loi pour la confiance dans l'économie numérique" (15 janvier 2003), en ligne : Assemblée nationale <http://www.assemblee-nationale.fr/12/projets/ pl0528.asp>. Il est possible d'y lire, à propos du titre III relatif à la sécurité, que : "Le commerce électronique permet de mettre en relation directe, dans des conditions totalement nouvelles, le vendeur et le consommateur final. Cependant son développement suppose la confiance des utilisateurs. Un des moyens d'augmenter cette confiance est l'utilisation de moyens de cryptographie qui permettent d'assurer des fonctions de signature électronique sécurisée, d'intégrité et de confidentialité des échanges".

36. Pour une appréciation des mécanismes de responsabilité, voir notamment les actes d'un colloque faisant suite à l'arrêt Swissair rendu le 15 novembre 1994 par le Tribunal fédéral suisse créant le principe de responsabilité fondé sur la confiance : Christine Chappuis et Bénédict Winiger, La responsabilté fondée sur la confiance. Vertrauenshaftung, Zurich, Schulthess Juristische Medien SA, 2001. 
en tant que "mécanisme particulier de régulation des différentes transactions qu'entretient l'entreprise avec ses différents partenaires»37. Pour ce faire, d'une part, les fondements définitionnels et les catégorisations possibles de la confiance seront examinés et, d'autre part, les facteurs favorisant la rencontre des droits et obligations des émetteurs et des destinataires de la confiance seront considérés.

\section{La confiance, une notion aux multiples fondements définitionnels}

Aujourd'hui, tout comme hier et certainement demain, la confiance est une notion qui fascine tant elle est modulable. Elle s'applique aux différentes disciplines des sciences humaines et sociales. Elle intéresse l'ensemble des activités de la vie politique, économique et sociale. La confiance est, en ce sens, une notion caméléon. Définir la notion de confiance n'est donc pas une chose facile. Il s'agit, entre autres, comme le souligne Servet d' 'une sorte de mot couvert ou de mot couvercle»38, comme le note Arrow d'une "institution invisible»39, comme l'écrivent Pallas et Richard d'"un concept flou, difficilement appréhendable et mesurable»40. Par conséquent, la confiance est généralement présentée au regard de ses fondements, de ses effets.

37. Voir notamment, Gérard Charreaux, "Le rôle de la confiance dans le système de gouvernance des entreprises" dans Francis Aubert et JeanPierre Sylvestre, dir., Confiance et rationalité, Paris, INRA Editions, 2001, 81 [Charreaux, "Gouvernance entreprise»].

38. Jean-Michel Servet, "Le chapeau" dans Philippe Bernoux et Jean-Michel Servet, dir., La construction sociale de la confiance, Paris, Montchrétien, 1997, 17 à la p. 17 [Servet, "Le chapeau"].

39. Kenneth J. Arrow, The Limits of Organization, New York / Londres, Norton, 1974 à la p. 26.

40. Valérie Pallas et Chrystelle Richard, "La confiance, créatrice de valeur?" dans Jean-Pierre Bréchet, dir., Valeur, marché et organisation, XIVe Journées nationales des I.A.E., Nantes, Presses académiques de l'Ouest, 1998. 
C'est pourquoi au lieu de se plier à un exercice définitionnel, certains auteurs ont préféré regrouper l'ensemble des facettes de cette notion en considérant que :

La confiance est un concept pluridimensionnel (un "hyperconcept", a-t-il été dit) et, de ce fait, déconcertant: il caractérise, simultanément, une relation et l'espoir fondé sur cette relation; un état donné de cette relation et une action (ou un mouvement); une attitude et un comportement; une propriété (ou une faculté) et une croyance; une norme sociale et un calcul; le produit d'une interaction et un arrangement institutionnel; un rapport et une ressource; une institution et une interaction; un engagement et une routine, etc. Catégorie plurielle, donc, et qui déroute l'analyse sociale : il s'agit en effet, à la fois : d'un état social préexistant, préalable à l'engagement d'une relation durable [...]; - mais aussi d'une situation construite au cours de l'action [...]; - ou d'un objectif recherché pour son potentiel de sécurité ontologique [...]; - ou, encore, d'une forme relationnelle, opposable aux autres. ${ }^{41}$

De plus, l'exercice définitionnel n'est pas aisé car il n'existe pas une mais plusieurs approches de la confiance qui, au demeurant, est un terme unique en français contrairement à l'anglais qui en possède deux: trust et confidence. Cette distinction est souvent mise de l'avant par les auteurs qui s'intéressent à la notion ${ }^{42}$. Ainsi, on peut lire que :

[I]l est important de souligner que là où le français ne dispose que d'un terme, l'anglais en possède deux : trust et confidence. La séparation n'est pas toujours simple à établir entre l'apport linguistique germanique et l'apport latin. Toutefois, trust paraît beaucoup plus fort que confidence car il inclut l'idée de faith, de foi, de croyance, alors que confidence [...] traduit une évaluation beaucoup plus

41. Thuderoz, "Introduction au propos", supra note 21 aux pp. 23 et 24 .

42. Voir notamment, Nikhlas Luhmann, "Familiarity, Confidence, Trust: Problems and Alternatives" dans Diego Gambetta, dir., Trust. Making and Breaking Cooperative Relations, Oxford, Basil Blackwell, 1988, 95 à la p. 97. 
objective des capacités à remplir une obligation et à établir une relation. ${ }^{43}$

Ou encore quant au fait de savoir ce que renferment les expressions "faire confiance" et "avoir confiance»44, on peut lire que :

[L]'anglais permet de séparer les deux idées, si on le souhaite, grâce à l'existence de deux mots au lieu d'un seul en français. Ainsi, pourra-t-on réserver le mot "trust" pour l'idée exprimée dans "faire confiance», se référant à une décision, et le mot "confidence" pour celle correspondant à "avoir confiance", se référant à un état cognitif, par exemple une probabilité; le mot "trustworthy" signifiant, avec une connotation plus neutre que cette expression française, "digne de confiance». 45

Nonobstant cette précision, l'expression "confiance" vient du mot latin confidentia exprimant l'espoir, l'assurance, ou encore l'audace ${ }^{46}$. Partant de là, le Petit Larousse ${ }^{47}$ nous enseigne que la confiance est le "sentiment de sécurité d'une personne qui se fie à quelqu'un, à quelque chose». Seule ici sera retenue l'idée de confiance s'établissant entre deux personnes, entre deux entités juridiques : l'émetteur et le destinataire de la confiance.

43. Servet, "Le chapeau", supra note 38 à la p. 26.

44. Au sujet de ces deux éléments de la confiance, il est possible de voir notamment: Raymond Boudon, Philippe Besnard et Mohamed Cherkaoui, dir., Dictionnaire de sociologie, Paris, Larousse, 2003 [Boudon, Besnard et Cherkaoui, "Dictionnaire de sociologie»].

45. Pierre Salmon, "Accumulation et destruction de la confiance : un schéma d'inspiration poppérienne" dans Francis Aubert et Jean-Pierre Sylvestre, dir., Confiance et rationalité, Paris, INRA Editions, 2001, 31 à la p. 33. Dans le même ouvrage, il est également possible de consulter à ce sujet : Francis Aubert et Jean-Pierre Sylvestre, "Introduction" dans Francis Aubert et Jean-Pierre Sylvestre, dir., Confiance et rationalité, Paris, INRA Editions, 2001, 7 à la p. 7 [Aubert et Sylvestre, "Introduction"].

46. Servet, "Le chapeau", supra note 38 à la p. 25.

47. Le Petit Larousse Illustré, 2004. 
Ce sentiment de sécurité invite l'émetteur de la confiance à s'inscrire dans un état de dépendance ${ }^{48}$. En effet, comme le précise le Dictionnaire d'éthique et de philosophie morale 49 , "faire confiance à une personne, c'est se placer dans un état de dépendance, ou prolonger un état de dépendance, à l'égard de la compétence et de la bonne volonté de cette personne; c'est accepter d'être vulnérable et admettre que le dépositaire de sa confiance exerce un pouvoir sur soi, ou sur quelque chose d'important pour soi»50. Et, il y est indiqué qu'en dehors de toute référence théologique, cette notion "reste étroitement liée, à certaines distinctions près, au fait de croire en la parole d'autrui et d'espérer que les pouvoirs supérieurs ne seront pas exercés à notre détriment»51. La confiance présuppose donc une forme d'abandon, de vulnérabilité52.

Toutefois, pour éviter toute forme d'abus, l'émetteur de la confiance doit demeurer vigilant. La confiance ne doit pas être aveugle ${ }^{53}$. Elle ne doit pas s'apparenter à un blanc-seing accordé à son destinataire ${ }^{54}$. Elle nécessite donc une part d'objectivité ${ }^{55}$.

48. Cet état de dépendance peut exister non seulement dans des relations de droit privé, mais aussi dans celles de droit public, soit entre les citoyens et l'État. À ce sujet, voir notamment, Sylvia Calmes, Du principe de protection de la confiance légitime en droits allemand, communautaire et français, Paris, Dalloz, 2001 [Calmes, "Protection"].

49. Monique Canto-Sperber, dir., Dictionnaire d'éthique et de philosophie morale, Paris, PUF, 2004 [Canto-Sperber, "Dictionnaire éthique»].

50. Ibid. s.v. "Confiance".

51. Ibid.

52. Sur cette idée de vulnérabilité, la définition de Mayer, Davis et Schoorman citée dans Charreaux, "Gouvernance entreprise», supra note 37 à la p. 82 .

53. Voir notamment, Bart Nooteboom, "Apprendre à faire confiance" dans Albert Ogien et Louis Quéré, dir., Les moments de la confiance. Connaissance, affects et engagements, Paris, Economica, 2006, 63 à la p. 68.

54. Aubert-Sylvestre, "Introduction", supra note 45 à la p. 8.

55. Alain Chirez, De la confiance en droit contractuel, thèse de doctorat, Université de Nice, 1977 [non publiée] [Chirez, "Droit contractuel»]. Selon l'auteur: "Il serait vain de prétendre faire de la confiance le produit systématique et prévisible de certaines données. La confiance ne se commande ni ne se requiert; elle s'éprouve et se donne. Pourtant ce 
Ainsi, en adoptant une attitude objective, la personne qui donne sa confiance conserve un sens critique qui lui permettra de retirer celle-ci à tout moment. La confiance n'est donc pas une chose acquise $^{56}$. Elle peut se transformer en méfiance ou en défiance ${ }^{57}$.

Partant de là, certains auteurs ont décrit la confiance comme étant "la condition d'un équilibre, elle fait l'alliance, un équilibre dans une stabilité que l'on veut faire durer. La confiance témoigne aussi du statut d'aliénation du sujet, elle engage le devenir, or il n'y a pas de devenir sans altérité, [...]. La confiance est donc le signe de l'aliénation, dans l'intersubjectivité et dans l'avenir, le devenir»58.

En disant que la confiance est le fruit d'un équilibre, d'interactions, on entend par là que la confiance peut provenir de relations durables 59 , de la réputation ${ }^{60}$, des émotions, des sentiments ${ }^{61}$, comme le prétendent notamment

sentiment n'est pas arbitraire. La confiance se paie et ceci, déjà, constitue un signe, car on n'achète pas le caprice. Ainsi, [...] la garantie due par le vendeur, qui entre pour une bonne part dans la fiabilité du produit vendu, se reporte sur le prix de vente. La confiance a un aspect comptable; donc elle se mesure et se pèse. [...] Notion purement subjective, la confiance repose sur des faits objectifs.", aux pp. 143 et 144

56. Peyrefitte, "Développement", supra note 34 à la p. 402 .

57. Jean-Michel Servet, "Paroles données : le lien de confiance» (1994) 4 Revue du Mouvement anti-utilitariste en sciences sociales 37 aux pp. 48 et $\mathrm{s}$.

58. Jean-Marie Thiveaud, «Avant-propos. Des formes complexes de la confiance : confiance, alliance, pacte, serment, etc." dans Philippe Bernoux et Jean-Michel Servet, dir., La construction sociale de la confiance, Paris, Montchrétien, 1997, 5 à la p. 7 [Thiveaud, "Formes complexes"].

59. Au sujet des relations durables et, donc de leur stabilité voir notamment, Thiveaud, "Formes complexes", supra note 58; Chirez, "Droit contractuel», supra note 55 aux pp. 225 à 248.

60. Voir notamment, Russell Hardin, "Communautés et réseaux de confiance" dans Albert Ogien et Louis Quéré, dir., Les moments de la confiance. Connaissance, affects et engagements, Paris, Economica, 2006, 89 à la p. 101 .

61. Chirez, "Droit contractuel», supra note 55 aux pp. 249 à 264. 
(2007) 37 R.D.U.S.

La confiance,

instrument de régulation

des environnements électroniques

Kreps $^{62}$, Orléan ${ }^{63}$. Elle peut également s'inscrire dans le cadre de réseaux sociaux, comme l'expliquent, entre autres, Gambetta64, Fukuyama65, Castells66, ou encore de relations contractuelles, comme le laisse entendre Chirez67, par exemple. Dès lors, ces interactions ne sont pas forcément basées sur un calcul68 comme le laissent entendre certains auteurs, Coleman ${ }^{69}$, Williamson ${ }^{70}$, qui considèrent que l'emploi de cette notion est galvaudée dès lors que

62. David M. Kreps, "Corporate Culture and Economic Theory" dans James E. Alt et Kenneth A. Shepsle, dir., Perspectives on positive political economy, Cambridge, Cambridge University Press, 1990, 90 [Kreps, "Corporate"]. Pour une illustration de la théorie de Kreps, à savoir la "confiance-réputation", voir notamment Lucien Karpik, "Préface. Les fondements symboliques de la confiance" dans Vincent Mangematin et Christian Thuderoz, dir., Des mondes de confiance. Un concept à l'épreuve de la réalité sociale, Paris, CBRS Editions, 2003, 7 à la p. 8 [Karpik, "Fondements symboliques"].

63. André Orléan, "Sur le rôle respectif de la confiance et de l'intérêt dans la constitution de l'ordre marchand" (1994) 4 Revue du Mouvement antiutilitariste en sciences sociales 17.

64. Diego Gambetta, Trust. Making and Breaking Cooperative Relations, Oxford, Basil Blackwell, 1988. Voir également, Bénédicte Reynaud, "Les conditions de la confiance. Réflexions à partir du rapport salarial" dans Francis Aubert et Jean-Pierre Sylvestre, dir., Confiance et rationalité, Paris, INRA Editions, 2001, 45.

65. Fukuyama, "Confiance et puissance», supra note 34.

66. Manuel Castells, La galaxie Internet, Paris, Fayard, 2001 [Castells, "Galaxie Internet"].

67. Chirez, "Droit contractuel», supra note 55. Voir également, Karim Medjad, "Confiance et relations contractuelles : frontière sémantique et frontière géographique" dans Romain Lauffer et Magali Orillard, dir., La confiance en question, Paris/Montréal, L'Harmattan, 2000, 117.

68. Parmi les auteurs en opposition avec cette position, voir notamment: Hélène Vérin, "Jalons pour une histoire de la confiance" dans Romain Laufer et Magali Orillard, dir., La confiance en question, Paris/Montréal, L'Harmattan, 2000, 37.

69. James S. Coleman, "Retations of Trust" dans James S. Coleman, dir., Foundations of Social Theory, Cambridge, Harvard University Press, 1990, 91. Pour une illustration de la théorie de Coleman, à savoir la "confiancecalcul", voir notamment Karpik, "Fondements symboliques", supra note 62 à la p. 8; Boudon- Besnard-Cherkaoui, "Dictionnaire de sociologie", supra note 44 .

70. Oliver E. Williamson, «Calculativeness, Trust, and Economic Organization" (1993) 36 J.L. \& Econ. 453. 
l'action d'entrer dans une relation n'est que le résultat d'un calcul entre gains escomptés et risques encourus ${ }^{71}$.

De ces trois positions découlent des définitions, des acceptions différentes de la notion de confiance. Sans rejeter totalement la "rationalité calculatoire" dans certaines situations ${ }^{72}$, nous sommes plus enclins à retenir les deux autres conceptions de la confiance et, plus particulièrement l'idée de communauté eu égard à notre problématique : savoir si la confiance peut être considérée comme un instrument de régulation des environnements électroniques.

La notion de communauté est souvent invoquée pour expliquer l'émergence d'une "nouvelle" forme de normativité encadrant les activités se déroulant sur Internet ${ }^{73}$ : code de conduite, politique de confidentialité, label de qualité, régulation par la technique. Ces règles qualifiées de spontanées, de "tricotées serrées" 74 , d'informelles, sont présentées, selon les cas, comme complétant ou suppléant les règles étatiques ${ }^{75}$.

71. Albert Ogien et Louis Quéré, Les moments de la confiance. Connaissance, affects et engagements, Paris, Economica, 2006 à la p. 1.

72. Pour une définition des notions "raison" et "rationalité", voir notamment Raymond Boudon et François Bourrigaud, dir., Dictionnaire critique de la sociologie, Paris, P.U.F., 2004 s.v. "raison" et "rationalité»; Encyclopédie de la philosophie, Paris, La Pochothèque, 2002 s.v. "raison" et "rationalité".

73. Pour une illustration de ce phénomène, voir notamment, Castells, "Galaxie Internet", supra note 66; Schultz, "eBay", supra note 7.

74. Trudel, Abran, Benyekhlef et Hein, "Droit du cyberespace", supra note 1 aux pp. 3-1 et s. Cette idée de communauté "tricotée serrée" s'apparente à la définition que donne l'Encyclopédie de la philosophie de la communauté en indiquant qu'il s'agit d'un "ensemble de sujets liés par un ou par plusieurs facteurs de différentes natures (ethnique, territoriale, linguistique, religieuse, économique, politique, etc.) qui les amènent à avoir plus de relations entre eux qu'avec les membres d'autres communautés".

75. Pour une illustration à l'égard de la protection des renseignements personnels dans le cadre du commerce électronique, voir notamment, Chassigneux, "Vie privée", supra note 12 aux pp. 175 et s. 
Cette situation s'explique par le fait, comme le souligne Fukuyama, que la communauté n'est :

[...] pas formée sur la base de règles et de règlements explicites, mais née d'un ensemble d'habitudes éthiques et d'obligations morales réciproques intériorisées par chaque membre de la communauté. Ce sont elles qui ont donné aux divers membres des raisons de se faire confiance. La décision de soutenir la communauté n'obéit donc pas à un intérêt économique étroit. ${ }^{76}$

Ou encore, comme l'explique Castells, au regard de la notion de réseaux que ceux-ci constituent :

[...] un ensemble de nœuds interconnectés. Ce type d'organisation humaine qui remonte à la nuit des temps entame une nouvelle vie à notre époque avec les réseaux de l'information, dont la force motrice est Internet. Les réseaux sont des modes d'organisation aux avantages extraordinaires, parce qu'ils sont naturellement flexibles et adaptables, qualités essentielles pour survivre et prospérer dans un environnement qui change vite. Et, l'auteur de continuer en disant qu'Internet permet aux réseaux [...] de démontrer ainsi leur aptitude naturelle à l'évolution tout en autorisant la coordination et la gestion de la complexité. D'où une association sans précédent de souplesse et d'efficacité, de prise de décision coordonnée et d'exécution décentralisée, d'expression personnelle et de communication horizontale planétaire, qui offre une forme d'organisation supérieure à l'activité humaine. ${ }^{77}$

Par conséquent, "la confiance est l'attente qui naît, au sein d'une communauté, d'un comportement régulier, honnête et coopératif, fondé sur des normes communément partagées, de la

76. Fukuyama, "Confiance et puissance", supra note 34 à la p. 20 .

77. Castells, "Galaxie Internet" supra note 66 aux pp. 9 et s. 
part des autres membres de cette communauté»78, étant entendu que ces normes ne sont pas forcément celles issues de l'État ${ }^{79}$.

Cette absence de référence à la puissance publique est souvent mise de l'avant pour illustrer le fait que ces règles ne constituent pas un ordre juridique car il n'existe pas de mécanismes de contrôle permettant, par exemple, de sanctionner adéquatement un manquement à la norme. Or, cette sanction doit-elle nécessairement être prononcée par l'autorité judiciaire? $\mathrm{Ne}$ peut-elle pas provenir d'autres entités 80 étant entendu que "chaque moyen de protection: juridique, autorégulation des firmes, surveillance de leurs politiques, usage des techniques de protection, a ses propres règles d'efficacité»81? Cette sanction ne peut-elle pas être induite de la notion de confiance?

78. Fukuyama, "Confiance et puissance», supra note 34 à la p. 36 .

79. Edward Lorenz, "Que savons-nous à propos de la confiance? Un tour d'horizon des contributions récentes" dans Vincent Mangematin et Christian Thuderoz, dir., Des mondes de confiance. Un concept à l'épreuve de la réalité sociale, Paris, CBRS Editions, 2003, 109 à la p. 112 (la confiance basée sur les normes) [Lorenz, "Confiance»]; Claude Giraud, "Les relations de confiance et la forme orale dans les échanges" dans Vincent Mangematin et Christian Thuderoz, dir., Des mondes de confiance. Un concept à l'épreuve de la réalité sociale, Paris, CBRS Éditions, 2003, 99.

80. Pour appuyer cette position, voir notamment : Lessig, "Code", supra note 16; Schultz, "eBay", supra note 7 à la p. 40. Pour une analyse de l'effectivité des codes de déontologie dans le monde des affaires, voir notamment: Geneviève Even-Granboulan, Éthique et économie, Paris Montréal, L'Harmattan, 1998 aux pp. 249 et s. (La morale et le droit des affaires : pourquoi des codes de déontologie?) ; Yves Poullet, "Vers la confiance. Vues de Bruxelles : un droit européen de l'Internet? Quelques considérations sur la spécificité de l'approche réglementaire européenne du cyberespace», allocution présentée lors du Colloque international Droit de l'Internet. Approches européennes et internationales, Paris, 19-20 novembre $2001<$ http://droit-internet-2001.univ-paris1.fr/pdf/vf/ Poullet_Y.pdf $>$ [Poullet, "Bruxelles"].

81. Louise Cadoux et Pierre Tabatoni, "Les défis d'Internet à la protection de la vie privée: institutions, marchés et techniques en Europe et aux États-Unis" dans Pierre Tabatoni, dir., La protection de la vie privée dans la société de l'information, Paris, PUF, 2000, 15 à la p. 20. 
À cet effet, il est possible de convenir qu'au même titre qu'elle se gagne, se donne, la confiance peut se perdre et être retirée. Cette remise en cause, ce déclin, voire ce retrait total de la confiance peut se produire lorsque le destinataire de la confiance ne respecte pas ses promesses ${ }^{82}$, ses engagements.

Dès lors, dans une relation contractuelle, il est possible de dire que "le contrat est un instrument de contrainte psychologique avant d'être un moyen de contrainte juridique»83. Ainsi, par exemple, quand une entreprise en ligne ne transmet pas toute l'information sur ses intentions en ce qui concerne la gestion des renseignements personnels ou les modifient en cours de contrat, on assiste à une perte de confiance de la part des internautes ${ }^{84}$. Cette attitude peut également conduire à des marques de défiance de la part des partenaires économiques et sociaux. On assiste alors à un déficit du "capital social» 85 ou encore de la réputation, ce qui s'apparente à une forme de sanction de la part des acteurs de la communauté.

82. Sur la corrélation entre promesse et responsabilité, voir notamment, Mohamed Nachi, Éthique de la promesse. L'agir responsable, Paris, P.U.F., 2003.

83. Chirez, "Droit contractuel", supra note 55 à la p. 82.

84. Pierre Tabatoni, "Stratégies sur les marchés d'information" dans Pierre Tabatoni, dir., La protection de la vie privée dans la société de l'information. L'impact des systèmes électroniques d'information, Cahiers des sciences morales et politiques, tome 1, Paris, P.U.F., 2000, 47. Dans ce chapitre, l'auteur expose p. 51 qu' "une bonne stratégie commerciale repose sur l'établissement de relations confiantes et durables avec les clients, qui, seules, permettent de rechercher en commun de meilleures solutions. Elle exige une politique dynamique et cohérente de communication. Trop souvent une promotion commerciale agressive met l'accent sur des procédures de surveillance (contrôle) des clients, avec un harcèlement qui accroît leur méfiance.".

85. L'idée de capital social a été mise de l'avant par Coleman. Voir à ce sujet, James S. Coleman, "Social Capital in the Creation of Human Capital» (1988) 94 American Journal of Sociology Supplement S95 
La confiance,

Or, la réputation, comme l'ont démontré Kreps et Orléan, est un facteur important de la confiance ${ }^{86}$. Il s'agit d'un sentiment commun que des individus éprouvent à l'égard d'une personne physique ou morale. Ce sentiment est basé sur la mémoire, étant entendu que la réputation s'établit au regard des expériences passées ${ }^{87}$. Ces dernières tiennent compte non seulement des expériences personnelles de l'émetteur de la confiance, mais aussi du phénomène de bouche à oreille. La mémoire est donc aussi bien individuelle que collective88. Partant de là, les internautes décident d'accorder, ou non, leur confiance à telle ou telle entreprise en ligne. La notion de réputation induit donc celle de loyauté de la part du commerçant électronique. Elle engendre également une forme de stabilité 89 , de fidélité 90 car, tout comme

86. La réputation constitue, avec la solvabilité, l'un des facteurs techniques de la confiance. Ainsi, "le pouvoir attractif de la réputation est une donnée importante du commerce et plus généralement des rapports des professionnels avec leur clientèle. Phénomène d'opinion publique, la réputation est d'abord l'oeuvre des profanes, ceux-là même qui propagent les rumeurs. La renommée du professionnel engendre chez le client une confiance d'autant plus forte qu'elle situe en quelque sorte la compétence à la hauteur de l'évidence. C'est à tout le moins un préjugé favorable que suscite la renommée du professionnel. Point n'est besoin de longues délibérations lors du choix du cocontractant ou de la marque du produit vendu, la confiance du créancier s'appuyant sur l'opinion des tiers", Chirez, "Droit contractuel», supra note 55 aux pp. 171 et 172.

87. Kreps, "Corporate», supra note 62 à la p. 107; Partha Dasgupta, "Trust as a commodity" dans Diego Gambetta, dir., Trust. Making and Breaking Cooperative Relations, Oxford, Basil Blackwell, 1988, 49.

88. "Il existe un troisième élément fondamental de la construction de la confiance : la mémoire, cette mémoire étant «individuelle» et collective. La confiance [...] est appuyée sur une connaissance, mais une connaissance qui n'est jamais totale, car la confiance serait alors inutile. Il existe donc un processus d'apprentissage de formation de cette mémoire "individuelle»; au niveau d'un groupe on peut citer l'exemple des antécédents communs qui vont donner les réseaux d'anciens élèves des grandes écoles, qui fournissent collectivement un réseau de confiance», Servet, "Le chapeau", supra note 38 à la p. 35.

89. Pour une illustration particulière de la notion de stabilité eu égard à la sécurité juridique, voir notamment, Calmes, "Protection", supra note 48.

90. Pour une application particulière de la notion, voir notamment, Patrick Simon, "Pour une meilleure compréhension de la fidélité à la marque : intégration des notions de confiance, de force de l'attitude et de force de 
Rome, la réputation ne se bâtit pas en un jour. Elle se construit avec le temps ${ }^{91}$. Ainsi :

La confiance que les clients portent au producteur-vendeur est le produit de la réputation que ce dernier a acquis, avec le temps, par le maintien de pratiques loyales. ${ }^{92}$

Cette appartenance à une communauté, cette réputation agissant aussi bien à titre de catalyseur que de couperet en cas de manquement, cette sécurité induite par la confiance, sont autant d'éléments qui, sans se référer nécessairement à un calcul ${ }^{93}$, permettent de définir cette notion qu'il est possible d'appréhender en terme de catégories.

\section{La confiance, une notion aux multiples catégories}

À l'image des éléments définitionnels, les tentatives de catégorisation de la notion sont nombreuses. Sans chercher à les analyser de façon exhaustive, notre propos est plutôt de présenter certaines relations s'établissant entre l'émetteur et le destinataire de la confiance. Cet exercice nous permettra de mieux comprendre la place accordée à la confiance dans un environnement où les acteurs ne sont pas en présence.

Encore plus que dans les relations contractuelles classiques, celles se faisant à distance ou entre absents nécessitent un important climat de confiance pour qu'il puisse $\mathrm{y}$

la marque. Une application aux produits de consommation courante", thèse de doctorat en sciences de gestion, Université Panthéon - Sorbonne (Paris I), 2002.

91. Pascale Trompette, "De la prudence ... à la confiance», dans Vincent Mangematin et Christian Thuderoz, dir., Des mondes de confiance. Un concept à l'épreuve de la réalité sociale, Paris, CBRS Editions, 2003, 99.

92. Karpik, "Fondements symboliques" supra note 62 à la page 8 .

93. Pour une analyse selon laquelle "la pensée rationnelle implique en ellemême une confiance primitive", voir : Jean-Claude Gens, "L'impératif oublié de la raison éclairée : rendre plus fort le discours de l'autre, c'està-dire aussi celui de la tradition" dans Francis Aubert et Jean-Pierre Sylvestre, dir., Confiance et rationalité, Paris, INRA Editions, 2001, 17. 
avoir un échange, une extériorisation de la volonté des parties. La confiance doit donc être inspirée et donnée. Dès lors, le destinataire et l'émetteur de cette marque de confiance se trouvent dans une situation soit horizontale, soit verticale ${ }^{94}$. Dans le premier cas, les deux protagonistes sont sur un pied d'égalité, ce sont des pairs. Dans le second cas, le destinataire est généralement dans une position de force par rapport à l'émetteur. Un sentiment de hiérarchie s'instaure entre les deux protagonistes. Dans le cadre des relations naissant sur Internet, il est alors possible de considérer que la confiance est de nature verticale. Ainsi, en donnant sa confiance, l'internaute se place sous l'autorité du commerçant électronique, il adhère aux conditions générales de vente, il accepte la politique de confidentialité sans pouvoir les discuter 95 . Ce type de relation illustre bien l'un des éléments constitutifs de la confiance, à savoir le lien de dépendance.

Partant toujours de cette idée d'extériorisation, certains auteurs considèrent la confiance de façon soit globale, soit objectivée ${ }^{96}$. Dans le premier cas, la situation est envisagée du côté

94. Jean-Michel Servet, "Paroles données : le lien de confiance", (1994) 4 Revue du Mouvement anti-utilitariste en sciences sociales semestrielle 37 [Servet, "Paroles données»]. Pour l'auteur, "deux types de confiance sont communément distinguées: verticale et horizontale. La confiance verticale est une relation hiérarchique entre subordonnés et supérieurs au sein d'une organisation ou d'une société. [...] La confiance horizontale est une relation entre semblables: individus occupant des fonctions similaires au sein d'une même unité de production ou dans des unités différentes", pp. 47-48.

95. Pour une appréciation de cette question, voir notamment: Chirez, "Droit contractuel", supra note 55 aux pp. 119 et s.

96. Éveline Baumann et François Leimdorfer, "La confiance? Parlons-en !" dans Philippe Bernoux et Jean-Michel. Servet, dir., La construction sociale de la confiance, Paris, Montchrétien, 1997, 359. Selon les auteurs, "la confiance est l'objectivation d'une posture interne au sujet, mais qui implique immédiatement une situation relationnelle. Cette dernière peut être un rapport du sujet vers le monde, une manière d'être, de faire ou de dire, elle peut être totale ou se limiter à une activité [...]. Dans de nombreux cas, elle est bien évidemment un rapport entre personnes. La confiance naît toujours dans un sujet animé, mais le 
de celui qui donne confiance : le commerçant électronique, par exemple. Il est alors fait référence à la crédibilité, à la transparence ${ }^{97}$, à l'information, à la sécurité, à la notoriété de l'entreprise. Ces éléments permettent, en principe, d'avoir confiance envers les biens et services proposés par l'entreprise. Dans le second cas, la situation s'appréhende du côté de celui qui donne sa confiance : l'internaute. Il est fait appel à son jugement qui peut être soit personnel ${ }^{98}$ ou individuel99, soit général100 ou organisationnel ${ }^{101}$ comme dans le cadre de système développé par eBay ${ }^{102}$.

Concernant la confiance organisationnelle, celle-ci peut être publique ou privée, étant entendu que cette dernière :

[...] se rapporte aux connaissances spécifiques que les individus ont accumulées sur telle ou telle organisation. Les

bénéficiaire de la confiance est à même de la susciter. Cette confiance peut être globale : "donner confiance" [...]. Mais elle peut aussi être objectivée : "donner sa confiance".", aux pp. 364 et 365.

97. "Au-delà de la légitimité des règles et de la croyance dans l'autre, la transparence entre ceux qui sont impliqués est ce qui permet de maintenir la confiance; transparence signifie ici un certain degré de savoir et d'information. La confiance est impossible si l'information est nulle; elle est inutile si l'information est parfaite et totale", Servet, "Paroles données", supra note 94 à la p.43; Poullet, "Bruxelles", supra note 80 .

98. Au sujet de ce type de confiance, voir notamment : Lorenz, "Confiance", supra note 79 à la p. 111 .

99. Concernant les bases individuelles de la confiance, voir notamment, Calmes, "Protection", supra note 48 aux pp. 359 et s.

100. Concernant les bases générales de la confiance faisant référence à la connaissance effective ou non de l'émetteur de la confiance des instruments normatifs encadrant une activité donnée, voir notamment Calmes, "Protection", supra note 48 aux pp. 360 et s.

101. Cécile Gode-Sanchez, "Confiance et proximité cognitive. Une analyse à partir du cas des organisations de défense», allocution présentée lors du 4th Congress on Proximity Economics: Proximity, Networks and Coordination, Université de la Méditerranée, Marseille, Juin 2004 <http://139.124.177.94/proxim/viewpaper.php?id=81> [Gode-Sanchez, "Confiance"].

102. Schultz, "eBay", supra note 7. 
individus ont une prise directe sur ces organisations en ce qu'ils participent à leur fonctionnement et leur développement. C'est le comportement des individus qui compose la structure institutionnelle et conditionne la production de confiance organisationnelle locale. ${ }^{103}$

À ce propos, on parle aussi de confiance intuitu personae basée sur ses propres expériences ou sur celles d'autrui à l'égard d'un même comportement ${ }^{104}$. Cette approche reprend la classification donnée par Luhmann ${ }^{105}$ et reprise par Giddens ${ }^{106}$ comme l'explique Lorenz en indiquant que la confiance peut être qualifiée d'interpersonnelle en opposition à celle dite systémique, soit le fait selon lequel :

[L]a confiance interpersonnelle se rapporte à la confiance placée dans des individus, ou un groupe d'individus, tandis que la confiance systémique qualifie celle, par exemple, placée dans le système juridique ou le système bancaire. La première suppose la croyance qu'une personne, ou un groupe de personnes, est digne de confiance; la seconde n'implique aucune croyance de ce genre. 107

Cette référence à l'individu et au système est particulièrement intéressante. En effet, même si ces deux pôles de

103. Gode-Sanchez, "Confiance", supra note 101.

104. Lorenz, "Confiance", supra note 79 à la p. 111; Servet, "Paroles données", supra note 94 aux pp. 45-47.

105. Pour Luhmann, une distinction s'établit entre la confiance assurée et celle qualifiée de décidée. Cette distinction dépend "de la perception et de l'attribution", "de notre capacité à distinguer dangers et risques", mais en aucun cas elle ne fait "référence à des questions de probabilité ou d'improbabilité" et elle "n'est pas un simple jeu à somme nulle, dans lequel plus il y a de confiance assurée moins la confiance décidée est requise et vice versa", Nikhlas Luhmann, "Confiance et familiarité. Problèmes et alternatives" dans Albert Ogien et Louis Quéré, dir., Les moments de la confiance. Connaissance, affects et engagements, Paris, Economica, 2006, 9 aux pp. 12 à 15. Pour une approche plus générale de la théorie luhmannienne de la confiance, il convient de se référer à l'ouvrage suivant : Luhmann, "Confiance», supra note 31.

106. Giddens, "Modernité", supra note 19.

107. Lorenz, "Confiance", supra note 79 à la page 110. 
la confiance peuvent être mis en opposition, il est également possible de considérer que ceux-ci sont interdépendants ou encore que l'un est le préalable de l'autre. Ainsi, dans le cadre des environnements électroniques, la confiance doit s'entendre non seulement dans la fiabilité des modes de transmission de l'information, mais aussi de l'interlocuteur. La confiance s'inscrit donc dans le temps. Elle doit exister avant, pendant et après la transaction. Pour ce faire, différents mécanismes sont mis en place, tant du côté de l'émetteur que du destinataire de la confiance, afin de la matérialiser.

\section{La confiance, une notion aux multiples matérialisations}

Si la confiance se donne, se perd, évolue avec le temps au fil des expériences, des relations, cela laisse supposer que cette dernière s'exprime de différentes façons. Que l'on pense aux instruments de nature normative ou contractuelle prescrivant les droits et obligations de tout un chacun et, visant à leur manière à (re)donner à la confiance ses lettres de noblesse ou en institutionnalisant cette dernière. Cette orientation s'illustre aussi bien dans le monde physique que sur Internet.

Eu égard à cet espace de communication, Schultz ${ }^{108}$ considère que la rematérialisation, la resocialisation et la rejudiciarisation sont des éléments agissant en ce sens, étant entendu que :

[L]a rematérialisation vise la mise en place, pour le commerce électronique, d'une architecture de contrôle, c'est-à-dire un ensemble d'outils technologiques de contrôle de l'identité et des activités des internautes [mais aussi des commerçants eu égard aux labels, aux codes de conduite et aux systèmes de résolution des litiges en ligne]. La resocialisation du commerce électronique a pour but de reformer sur Internet des communautés de confiance. [...] Une communauté numérique à base commerciale appelle

108. Schultz, "eBay», supra note 7. 
son propre système de résolution des litiges - ce qui correspond à la rejudiciarisation [...]. ${ }^{109}$

Concernant la labellisation, il convient de reconnaître que celle-ci, quelle soit interne ou externe ${ }^{110}$, favorise l'expression de la confiance du fait de la transparence développée par le destinataire à l'attention de l'émetteur de cette dernière. Il est donc important que les intentions du destinataire soient crédibles, claires, compréhensibles et accessibles. De plus, en l'espèce, l'émetteur doit s'entendre aussi bien de l'internaute lui-même, mais aussi des autorités de certification. En effet, les entreprises en ligne doivent recevoir la confiance d'une tierce partie pour (re)gagner celle des internautes. Ce mécanisme conduit à l'établissement d'une "relation triangulaire»111:

[L]e label de qualité est donc une image, un logo, un signe de reconnaissance qu'un marchand appose sur son site pour rassurer ses clients et notamment le plus démuni d'entre eux à savoir le consommateur. Une relation triangulaire est donc mise en place, conformément au processus même de certification, qu'il soit d'identité ou de qualité. En effet, il implique une autorité de certification, un abonné, c'est-à-dire un commerçant qui emploie les services de l'autorité de certification, et enfin l'utilisateur final, le consommateur, qui "magasine" sur la base de la confiance que le sceau lui apporte. ${ }^{112}$

En apposant le label de l'autorité de certification, l'entreprise en ligne adhère aux conditions émises par cette dernière. En effet, le commerçant électronique accepte, d'une part, que ses engagements en matière de protection des renseignements

109. Ibid. aux pp. 35 et $\mathrm{s}$.

110. Pour une analyse de la labellisation interne (politique de confidentialité) et externe (autorité de certification) quant à la protection des renseignements personnels, voir notamment : Chassigneux, "Vie privée", supra note 12 d'une part, pp. 178 et s. et, d'autre part, pp. 201 et s.

111. Vincent Gautrais, "La labellisation de qualité des sites Internet: un sésame voué à la sécurité du consommateur" (1999) 3 Ubiquité 91.

112. Ibid. à la p. 91 . 
personnels ou que ses mécanismes de sécurité soient soumis à l'examen d'un tiers et, d'autre part, que l'accréditation qui lui est accordée puisse lui être retirée en cas de manquement. L'adhésion, même si elle est volontaire, est donc conditionnelle au respect des règles mises en place par l'autorité de certification. Ainsi, en devenant titulaire d'un label, une entreprise en ligne gagnera en crédibilité aux yeux des internautes. Crédibilité qui fait référence aux notions de relation durable ou encore de réputation permettant de définir la notion de confiance.

Concernant les modes alternatifs de résolution des conflits offerts aux internautes, soit directement sur les sites commerciaux, soit sur ceux des autorités de certification ou encore de contrôle, il est permis de considérer que cette solution "contribue à la régulation par place de marché parce que les utilisateurs de celles-ci se tournent moins vers des lieux externes de règlements des différends, qui apportent habituellement leur inévitable lot d'influence normative»113. Ces mécanismes de contrôle $^{114}$ ont souvent été décriés car se situant en dehors de la contrainte étatique et, par conséquent ne faisant pas partie de l'ordre juridique.

Toutefois, comme le rappellent certains auteurs, dont Rocher, la reconnaissance d'un ordre juridique est conditionnée, entre autres, à l'existence d'agents ou d'appareils "reconnus dans l'unité sociale comme étant spécialisés pour: 1) élaborer de nouvelles règles ou modifier celles qui existent; 2) interpréter les règles existantes; 3) les appliquer et les faire respecter»115.

113. Schultz, "eBay", supra note 7 à la p. 38. Dans le même ordre d'idée, voir "Pistes pour renforcer la confiance dans le commerce électronique - Avis $\mathrm{n}^{\circ}$ 3" (Juin 2004), en ligne: Observatoire des droits de l'Internet $<$ http://www.internetobservatory.be/internet_observatory/pdf/advices/advice_fr_003.pdf> aux pp. 22 et s. [Observatoire, "Confiance»].

114. Pierre Trudel, "La Lex Electronica" dans Charles-Albert Morand, dir., Le droit saisi par la mondialisation, Bruxelles, Bruylant, 2001, 221 aux pp. 257 et s.; Poullet, "Bruxelles", supra note 79.

115. Rocher, "Sociologie», supra note 8 à la p. 104. 
Il est entendu que ces fonctions, d'une part, "peuvent être remplies par des agents ou des appareils différents, ou par les mêmes"116 et, d'autre part, que "les règles et les agents ou appareils doivent faire preuve de stabilité dans le temps, d'une relative permanence. Ces règles ne doivent pas sans cesse varier et les agents être constamment relayés»117. Les mécanismes développés, entre autres, par eBay ${ }^{118}$, Amazon ${ }^{119}$ ou encore par des organismes tels que TRUSTe ${ }^{120}$, ECODIR ${ }^{121}$ s'inscrivent dans cette démarche de surveillance par les pairs, par les membres de la communauté.

Il est donc important, comme le précise notamment l'Observatoire des droits de l'Internet ${ }^{122}$, que les consommateurs et les commerçants soient sensibilisés à ce processus dont la procédure doit être transparente, facilement accessible, rapide et peu onéreuse ${ }^{123}$. En effet, ces mécanismes conduisent au maintien ou au retrait de la confiance par les émetteurs de celle-ci selon la solution apportée à une situation donnée, mais aussi à la diligence pour résoudre le point litigieux.

Labellisation, modes alternatifs de résolution des conflits sont des outils qui permettent aux destinataires et aux émetteurs de la confiance de se rencontrer compte tenu de l'ouverture dont ils doivent faire preuve. Ouverture quant à la mise en place de tels mécanismes, mais aussi quant aux produits et services proposés, quant à la qualité du destinataire de la confiance, à sa responsabilité par rapport au cycle de vie du contrat comme prescrit, par exemple, dans la Loi concernant le cadre juridique des

\footnotetext{
116. Ibid.

117. Ibid.

118. En ligne : eBay <http://www.ebay.com>, voir notamment la section "Feedback Forum".

119. En ligne : Amazon <http://www.amazon.com>.

120. En ligne : Truste <http://www.truste.org>.

121. En ligne : Ecodir <http://www.ecodir.org $>$.

122. En ligne : Economie <http://www.internet-observatory.be>.

123. Observatoire, "Confiance", supra note 113 aux pp. 26 et 27.
} 
technologies de l'information ${ }^{124}$ ou la Loi pour la confiance dans l'économie numérique ${ }^{125}$. Ces lois indiquent, en effet, que le destinataire est responsable de la non-exécution des obligations contractuelles, de la réalisation d'une activité à caractère illicite, du bris de confidentialité du document dont il a la garde, sauf s'il démontre que cette situation est indépendante de sa volonté et qu'il a pris les moyens nécessaires pour minimiser les inconvénients qui en résultent.

Cette information ${ }^{126}$ peut provenir du destinataire de la confiance, ce qui est susceptible de renforcer son image à l'égard des tiers. Elle peut également être le fait des membres de la communauté cherchant à renseigner les internautes sur le sérieux ou non des activités d'une personne physique ou morale qui "propose ou assure à distance et par voie électronique la fourniture de biens et de services» ${ }^{127}$. On retrouve ici l'idée de bouche à oreille, de mémoire, de réputation favorisant l'établissement d'une confiance individuelle et collective nécessaire au bon déroulement des activités effectuées dans le cadre des environnements électroniques.

\section{Conclusion}

Même si les éléments définitionnels, les catégories et les matérialisations de la confiance sont multiples, nous avons voulu démontrer, d'une part, qu'à l'instar des instruments législatifs qui sont discutés avant d'être adoptés, la confiance s'établit au fil des expériences avant de s'installer. D'autre part, si les règles contenues dans les instruments législatifs doivent être appliquées, pour continuer à agir la confiance doit s'entretenir. Enfin, tout

124. Loi concernant le cadre juridique des technologies de l'information, L.R.Q., c. C-1.1, rticles $22,26,27,36$ et 37 .

125. Loi pour la confiance dans l'économie numérique, supra note 26 à l'article 15.

126. Pour une analyse du concept d'information, voir notamment : Vallée et Mackaay, "Confiance", supra note 32

127. Loi pour la confiance dans l'économie numérique, supra note 26 à l'article 14 alinéa $1^{\mathrm{er}}$. 
comme une amende ou l'annulation d'un contrat sont des sanctions répondant au non-respect d'une obligation contractuelle, la défiance, la méfiance, la mauvaise publicité, la désaffectation sont au nombre des conséquences d'un bris de confiance.

Dès lors, tout comme les sources institutionnelles et celles qui sont substantielles ${ }^{128}$, la confiance $\mathrm{du}$ fait de son établissement, de son maintien et de sa sanction par les acteurs, permet de réguler les environnements électroniques. Toutefois, la confiance est souvent orientée du côté de son destinataire et présentée comme étant synonyme de sécurité, de responsabilité. Elle ne peut cependant pas exister sans émetteur. En effet, le destinataire peut tout mettre en ouvre pour rendre son site Web attrayant, pour offrir les meilleurs produits et services, pour donner une information claire, lisible et facilement accessible, si l'émetteur n'est pas disposé à lui accorder sa confiance, tous les efforts resteront lettre morte. La confiance ne se commande pas, elle se donne et se reprend. Cette action est le fait de son émetteur agissant à titre individuel ou collectif. La confiance doit donc s'envisager non seulement du côté du destinataire, mais aussi de l'émetteur pour être un instrument de régulation effectif ${ }^{129}$ des environnements électroniques.

128. Trudel, "Lex Electronica", supra note 114 aux pp. 236 et s.

129. Pour une analyse générale de la notion d'effectivité, voir notamment, Guy Rocher, "L'effectivité du droit" dans Andrée Lajoie, Roderick A. Macdonald et Richard Janda, dir., Théories et émergence du droit: pluralisme, surdétermination et effectivité, Montréal/Bruxelles, Thémis/Bruylant, 1998, 133. 